\title{
Risk stratification of normotensive pulmonary embolism: prognostic impact of copeptin
}

\author{
Kristian Hellenkamp ${ }^{1}$, Johanna Schwung ${ }^{1}$, Heidi Rossmann², Anja Kaeberich ${ }^{3}$, \\ Rolf Wachter ${ }^{1,4}$, Gerd Hasenfuß ${ }^{1,4}$, Stavros Konstantinides ${ }^{3}$ and \\ Mareike Lankeit ${ }^{1,3}$
}

Affiliations: ${ }^{1}$ Clinic of Cardiology and Pneumology, Heart Centre, Georg-August University of Göttingen, Göttingen, Germany. ${ }^{2}$ Institute of Clinical Chemistry and Laboratory Medicine, University Medical Centre Mainz, Johannes Gutenberg-University Mainz, Mainz, Germany. ${ }^{3}$ Centre for Thrombosis and Hemostasis (CTH), University Medical Centre Mainz, Johannes Gutenberg-University Mainz, Mainz, Germany. ${ }^{4}$ Partner site Göttingen, German Cardiovascular Research Centre (DZHK), Göttingen, Germany.

Correspondence: Mareike Lankeit, Centre for Thrombosis and Hemostasis (CTH), University Medical Centre Mainz, Johannes Gutenberg-University of Mainz, Langenbeckstraße 1, 55131 Mainz, Germany.

E-mail: mlankeitauni-mainz.de

ABSTRACT The prognostic value of copeptin, the C-terminal fragment of the precursor protein of vasopressin which is released upon stress, and hypotension in pulmonary embolism is unknown, especially if combined with biomarkers reflecting different pathophysiological axes such as myocardial injury (highsensitivity troponin $\mathrm{T}(\mathrm{hs} \mathrm{TnT})$ ) and stretch (N-terminal pro-brain natriuretic peptide (NT-proBNP)).

We prospectively studied 268 normotensive pulmonary embolism patients included in a single-centre cohort study.

Patients with an adverse 30-day outcome (5.6\%) had higher copeptin levels than patients with a favourable course (median (interquartile range) 51.8 (21.6-90.8) versus $13.2(5.9-39.3) \mathrm{pmol} \cdot \mathrm{L}^{-1} ; \mathrm{p}=0.020$ ). Patients with copeptin levels above the calculated optimal cut-off value of $24 \mathrm{pmol} \cdot \mathrm{L}^{-1}$ had a 5.4 -fold increased risk for an adverse outcome (95\% CI 1.68-17.58; $\mathrm{p}=0.005)$. We developed a strategy for risk stratification based on biomarkers. None of 141 patients $(52.6 \%)$ with hsTnT $<14 \mathrm{pg} \cdot \mathrm{mL}^{-1}$ or NT-proBNP $<600 \mathrm{pg} \cdot \mathrm{mL}^{-1}$ had an adverse outcome (low risk). Copeptin $\geqslant 24 \mathrm{pmol} \cdot \mathrm{L}^{-1}$ stratified patients with elevated hsTnT and NT-proBNP as intermediate-low and intermediate-high risk $(5.6 \%$ and $20.0 \%$ adverse outcome, respectively). Compared to the algorithm proposed by the 2014 European Society of Cardiology guideline, more patients were classified as low risk $(52.8 \%$ versus $17.5 \%, \mathrm{p}<0.001)$ and more patients in the intermediate-high risk group had an adverse outcome (20.0\% versus $11.6 \%)$.

Copeptin might be helpful for risk stratification of normotensive patients with pulmonary embolism, especially if integrated into a biomarker-based algorithm.

$@$ ERSpublications

Copeptin may improve risk stratification of normotensive pulmonary embolism by reflecting a new pathophysiological axis http://ow.ly/R9nOV

For editorial comments see Eur Respir J 2015; 46: 1551-1553 [DOI: 10.1183/09031936.01562-2015]

This article has supplementary material available from erj.ersjournals.com

Received: June 012015 | Accepted after revision: July 152015 | First published online: Oct 222015

Support statement: This study was supported by the German Federal Ministry of Education and Research (BMBF 01EO1003). The authors are responsible for the contents of this publication. Funding information for this article has been deposited with FundRef.

Conflict of interest: None declared.

Copyright @ERS 2015 


\section{Introduction}

In normotensive patients with acute pulmonary embolism, risk stratification is mandatory to define the appropriate treatment strategy [1,2]. According to the current (2014) guideline of the European Society of Cardiology (ESC), risk assessment should begin with a validated clinical risk prediction score to distinguish between low and intermediate risk pulmonary embolism [1]. In patients classified at intermediate risk, further stratification into intermediate-low and intermediate-high risk groups should be based on imaging procedures for the assessment of right ventricular (RV) dysfunction and laboratory biomarkers. However, although clinical scores and combination models such as the Bova score $[3,4]$ were developed with the aim of identifying patients with elevated risk (intermediate-high risk), the identification of the patient subgroup that may benefit from more aggressive treatment strategies remains challenging [2].

RV dysfunction is considered to be the critical determinant of outcome in acute pulmonary embolism. The sudden increase of pulmonary artery pressure may initiate a vicious circle leading to decreased left ventricular preload and low cardiac output, resulting in systemic hypotension [1,5]. Vasopressin (also known as antidiuretic hormone or arginine vasopressin) is a highly conserved peptide that plays a key role in cardiovascular homeostasis [6]. It is primarily produced in the magnocellular neurons of the hypothalamus, released upon changes in plasma osmolality, stress and hypotension and induces arteriolar vasoconstriction and water reabsorption in the kidneys [6]. Since vasopressin is highly unstable in plasma with a short half-life time of $\sim 20 \mathrm{~min}$, copeptin, the inactive 39-amino-acid C-terminal fragment of the precursor protein of vasopressin, which is released in equimolar amounts with vasopressin, can be used as a surrogate biomarker with high stability in plasma [6]. Due to its fast release kinetics [7], copeptin emerged as a valuable diagnostic and prognostic biomarker in several acute cardiovascular diseases [8-10] and conditions [11] allowing therapeutic decision making at the moment of presentation. In acute pulmonary embolism, copeptin might reflect a novel pathophysiological axis of pulmonary embolism severity by indicating the systemic response to impaired haemodynamics due to RV dysfunction and failure. Thus, combination with other biomarkers for risk assessment of acute pulmonary embolism reflecting either myocardial injury (such as high-sensitivity troponin $\mathrm{T}(\mathrm{hs} \operatorname{TnT})[1,12,13]$ ) or myocardial stretch (such as $\mathrm{N}$-terminal pro-brain natriuretic peptide (NT-proBNP) $[1,14]$ ) appears to be especially useful.

The aim of the present study was to investigate the prognostic impact of copeptin reflecting a new pathophysiological axis for risk stratification of normotensive pulmonary embolism patients.

\section{Material and methods}

Study subjects and study design

We prospectively studied consecutive normotensive patients aged $\geqslant 18$ years with objectively confirmed acute symptomatic pulmonary embolism at the University Hospital of Göttingen (Göttingen, Germany), who were included in an ongoing noninterventional cohort study (Pulmonary Embolism Registry of Göttingen) between September 2008 and August 2014. The study protocol has been described previously [12, 15] and details and definitions are provided in the online supplementary material. Treatment decisions were made by the physicians caring for the patient according to the current ESC guideline [16] and not influenced by the study protocol. Study results were not communicated to the clinicians and thus not used to guide patient management or to monitor the effects of treatment during the hospital stay or at any time during the 30-day follow-up period. The study was conducted in accordance with the amended Declaration of Helsinki, the study protocol was approved by the local independent ethics committee of the Medical University Göttingen (Göttingen, Germany) and all patients gave written informed consent for their participation in the study.

The primary outcome of the study was an adverse 30-day outcome, defined as pulmonary embolism-related death or at least one of the following complications: 1) need for intravenous catecholamine administration; 2) mechanical ventilation; and 3) cardiopulmonary resuscitation. All-cause death within 30 days was defined as secondary outcome. Causes of death were independently adjudicated by review of the medical records and autopsy reports (if performed) by three of the contributing authors (K. Hellenkamp, J. Schwung and M. Lankeit). Death was determined to be pulmonary embolism-related if it was 1) confirmed by autopsy; 2) followed a clinically severe episode of acute pulmonary embolism; and 3) in the absence of an alternative diagnosis.

\section{Laboratory biomarker testing}

Venous blood samples were collected on admission and after $24 \mathrm{~h}$ and immediately stored at $-80^{\circ} \mathrm{C}$. Plasma levels of copeptin, hsTnT and NT-proBNP were measured as described in the online supplementary material.

\section{Statistical analysis}

Using the modified Kolmogorov-Smirnov test (Lilliefors test) the continuous variables tested in this study were found not to follow a normal distribution. They are therefore presented as median (interquartile 
range (IQR)). Comparison of continuous variables was performed using the Mann-Whitney U-test and paired variables were compared using the Wilcoxon test. For comparisons of categorical variables Fisher's exact test was used. To test for correlations between continuous variables the Spearman $\rho$ coefficient was calculated. Additionally, the relationship between variables and copeptin concentrations above the median was tested using univariable logistic regression analysis and results presented as OR (95\% CI). The area under the curve (AUC) of copeptin, hsTnT and NT-proBNP with regard to study outcomes was determined using receiver operating characteristic (ROC) analysis and comparison performed using the test of de Long, de Long and Clarke-Pearson. Youden-index quantification was used to identify the optimal copeptin cut-off values for prediction of study outcomes. Specificity, sensitivity, negative and positive predictive values and the negative and positive likelihood ratios were calculated for clinical sores, biomarkers and combinations of biomarkers. Dichotomisation of the Bova score and the ESC 2014 risk categories were performed by combining patients stratified as low risk and intermediate-low risk (tested versus patients classified as intermediate-high risk). To compare the distribution of patients in risk classes by the use of different risk assessment strategies, the McNemar-Bowker test was used. The prognostic relevance of comorbidities, haemodynamic, clinical and laboratory findings as well as risk assessment models with regard to study outcomes were estimated using univariable logistic regression analysis. The independence of the prognostic value of copeptin levels above the calculated cut-off value with regard to the primary outcome was tested in multivariable logistic regression models which each parameter identified as a predictor of copeptin concentrations above the median (online supplementary table S1) separately. Additionally, parameters univariably associated with an adverse 30-day outcome were included in a multivariable logistic regression model using backward stepwise selection.

All statistical tests used in this study were two-sided and used a significance level of 0.05 . Analyses were performed using SPSS (version 22.0; SPSS Inc., Chicago, IL, USA).

\section{Results}

\section{Baseline study findings}

Overall, 268 normotensive patients with confirmed pulmonary embolism were included in the study. Figure 1 shows the flow chart of patient enrolment and exclusion. The medical history and baseline characteristics of the study patients are summarised in table 1. The imaging procedures for confirmation of pulmonary embolism are described in the online supplementary material. In 255 patients (95.1\%) RV function could be assessed using diagnostic multidetector computed tomography (MDCT); of those, 111 patients (43.5\%) were diagnosed with RV dysfunction. An echocardiographic examination was performed within 48 h in 157 patients (58.6\%); of those, 77 patients (49.0\%) were diagnosed with RV dysfunction. Of note, 56 patients had evidence of RV dysfunction on both echocardiography and MDCT and in four patients assessment of RV function was neither performed on MDCT nor on echocardiography.

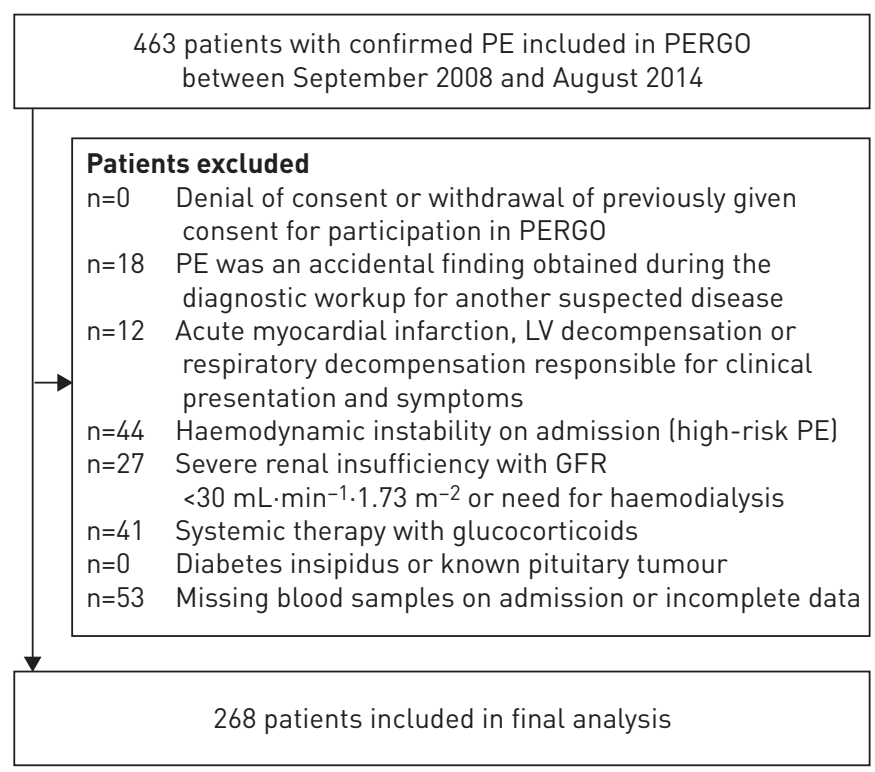

FIGURE 1 Flow chart of patient enrolment and exclusion. Definitions are provided in the material and methods section of the online supplementary material. PE: pulmonary embolism; PERGO: Pulmonary Embolism Registry of Göttingen; LV: left ventricular; GFR: glomerular filtration rate. 
TABLE 1 Medical history and baseline characteristics of the study patients

\begin{tabular}{|c|c|c|c|c|}
\hline & All study patients & Copeptin $<14 \mathrm{pmol} \cdot \mathrm{L}^{-1}$ & Copeptin $\geqslant 14 \mathrm{pmol} \cdot \mathrm{L}^{-1}$ & p-value \\
\hline Subjects $n$ & 268 & 134 & 134 & \\
\hline Male & $128(47.8)$ & $58(43.3)$ & $70(52.2)$ & 0.178 \\
\hline Age years & $70.5(53-77)$ & $62.5(46-74)$ & $72.5(64-79)$ & $<0.001$ \\
\hline BMI $\mathrm{kg} \cdot \mathrm{m}^{-2}$ & $27.8(24.8-31.2)$ & $28.0(25.0-32.1)$ & $27.6(24.5-30.6)$ & 0.300 \\
\hline \multicolumn{5}{|l|}{ Risk factors for VTE } \\
\hline History of VTE & $85 / 267$ (31.8) & $50 / 133(37.6)$ & $35(26.1)$ & 0.049 \\
\hline Previous trauma/surgery ${ }^{\#}$ & $55(20.5)$ & $28(20.9)$ & $27(20.1)$ & 1.000 \\
\hline Travel/immobilisation ${ }^{\#}$ & $67(25.0)$ & $31(23.1)$ & $36(26.9)$ & 0.573 \\
\hline Pregnancy/post-partum? & $1(0.4)$ & $1(0.7)$ & $0(0)$ & 1.000 \\
\hline Unprovoked $\mathrm{PE}^{+}$ & $160(59.7)$ & 78 (58.2) & $82(61.2)$ & 0.709 \\
\hline \multicolumn{5}{|l|}{ Comorbidities } \\
\hline Malignant tumour $\S$ & $37(13.8)$ & 15 (11.2) & $22(16.4)$ & 0.288 \\
\hline Chronic cardiopulmonary disease & 93 (34.7) & $35(26.1)$ & 58 (43.3) & 0.005 \\
\hline Diabetes mellitus & 49 (18.3) & 15 (11.2) & $34(25.4)$ & 0.004 \\
\hline Anaemia & $91(34.0)$ & $41(30.6)$ & 50 (37.3) & 0.302 \\
\hline Renal insufficiency & 73 (27.2) & $23(17.2)$ & 50 (37.3) & $<0.001$ \\
\hline \multicolumn{5}{|c|}{ Symptoms and clinical status on admission } \\
\hline Symptom onset $<24 \mathrm{~h}$ & $130(48.5)$ & $54(40.3)$ & $76(56.7)$ & 0.010 \\
\hline Chest pain & $155 / 267(58.1)$ & $86 / 133(64.7)$ & 69 (51.5) & 0.035 \\
\hline Dyspnoea & $233 / 267(87.3)$ & $116 / 133$ (87.2) & 117 (87.3) & 1.000 \\
\hline Syncope & $32(11.9)$ & $7(5.2)$ & $25(18.7)$ & 0.001 \\
\hline Systolic blood pressure $\mathrm{mmHg}$ & $130(120-150)$ & $130(120-150)$ & $130(120-141)$ & 0.017 \\
\hline Mild hypotension & $19 / 262(7.3)$ & $6 / 130(4.6)$ & $13 / 132(9.8)$ & 0.152 \\
\hline Heart rate bpm & $88(74.25-105)$ & $84(75.5-97.5)$ & $91(73.5-110.0)$ & 0.042 \\
\hline Tachycardia & $80 / 264$ (30.3) & $31 / 132$ (23.5) & 49/132 (37.1) & 0.022 \\
\hline Hypoxia & $62 / 224(27.7)$ & $21 / 107(19.6)$ & $41 / 117$ (35.0) & 0.011 \\
\hline RV dysfunction on echocardiography & $77 / 157(49.0)$ & $29 / 69(42.0)$ & $48 / 88$ (54.5) & 0.148 \\
\hline RV dysfunction on MDCT & $111 / 255(43.5)$ & $45 / 130(34.6)$ & $66 / 125$ (52.8) & 0.004 \\
\hline \multicolumn{5}{|l|}{ Laboratory biomarkers } \\
\hline $\mathrm{hsTnT} \mathrm{pg} \cdot \mathrm{mL}^{-1}$ & $24.6(8.6-57.9)$ & $11.2(4.9-43.8)$ & $36.1(15.1-73.7)$ & $<0.001$ \\
\hline $\mathrm{hsTnT} \geqslant 14 \mathrm{pg} \cdot \mathrm{mL}^{-1}$ & $166 / 266(62.4)$ & $61 / 132(46.2)$ & $105 / 134(78.4)$ & $<0.001$ \\
\hline NT-proBNP pg.mL ${ }^{-1}$ & $687(122-2564)$ & $309(74-1640)$ & $1238(290-3775)$ & $<0.001$ \\
\hline $\mathrm{NT}-$ proBNP $\geqslant 600 \mathrm{pg} \cdot \mathrm{mL}^{-1}$ & $138 / 267(51.7)$ & $55 / 134(41.0)$ & $83 / 133(62.4)$ & 0.001 \\
\hline \multicolumn{5}{|l|}{ Risk assessment models } \\
\hline \multicolumn{5}{|l|}{ ESC 2014 algorithm } \\
\hline Low & 47 (17.5) & $41(30.6)$ & $6(4.5)$ & $<0.001$ \\
\hline Intermediate-low & $135(50.4)$ & $63(47.0)$ & 72 (53.7) & 0.328 \\
\hline Intermediate-high & $86(32.1)$ & $30(22.4)$ & $56(41.8)$ & 0.001 \\
\hline \multicolumn{5}{|l|}{ Bova score $e^{f}$} \\
\hline Low & $130(48.7)$ & $84(62.7)$ & $46(34.6)$ & $<0.001$ \\
\hline Intermediate-low & $93(34.8)$ & $41(30.6)$ & $52(39.1)$ & 0.159 \\
\hline Intermediate-high & $44(16.5)$ & $9(6.7)$ & 35 (26.3) & $<0.001$ \\
\hline
\end{tabular}

Data are presented as $\mathrm{n}, \mathrm{n}(\%)$, median (interquartile range) or $\mathrm{n} / \mathrm{N}(\%)$, unless otherwise stated. Risk factors, comorbidities, symptoms, haemodynamics, laboratory values and PE severity classification in all study patients and according to the median copeptin level are presented. BMI: body mass index; VTE: venous thromboembolism; PE: pulmonary embolism; bpm: beats per minute; RV: right ventricular; MDCT: multidetector computed tomography; hsTnT: high-sensitivity troponin T; NT-proBNP: N-terminal pro-brain natriuretic peptide; ESC: European Society of Cardiology. ${ }^{\#}$ : within the past 4 weeks; ${ }^{\text {ๆ }}$ : 6 weeks after delivery; ${ }^{+}$: defined as absence of a temporary or reversible risk factor such as surgery, trauma, immobilisation, travel, contraception/hormone replacement therapy or pregnancy; ${ }^{\S}$ : defined as active or under treatment within the past 6 months; ${ }^{f}$ : one patient could not be classified using the Bova score due to missing data. Definitions are provided in the material and methods section of the online supplementary material.

Initial treatment of the study patients consisted of either anticoagulation with unfractionated heparin or low molecular weight heparin/fondaparinux at weight-adjusted dosage and 29 patients (10.8\%) received reperfusion therapy. Of those, 17 patients were included in the Pulmonary Embolism Thrombolysis (PEITHO) study [17] and randomised to single-bolus tenecteplase versus placebo, 12 patients received early systemic thrombolysis and two patients included in the PEITHO study additionally underwent surgical embolectomy.

Copeptin plasma levels on admission ranged between 0.9 and $705.0 \mathrm{pmol} \cdot \mathrm{L}^{-1}$ with a median (IQR) concentration of $13.7(5.9-44.5) \mathrm{pmol} \cdot \mathrm{L}^{-1}$. We observed a weak correlation of copeptin concentrations with 
age $(r=0.314, p<0.001)$, NT-proBNP $(r=0.318, p<0.001)$ and hsTnT $(r=0.363, p<0.001)$ and a weak inverse correlation with glomerular filtration rate $(\mathrm{r}=-0.308, \mathrm{p}<0.001)$. Differences in medical history and baseline characteristics of study patients with copeptin concentrations below the median compared to patients with copeptin above the median are shown in table 1. Additionally, as shown in online supplementary table S1, a number of parameters were associated with copeptin concentrations above the median.

To further investigate the kinetics of copeptin, we measured copeptin concentration in blood samples collected $24 \mathrm{~h}$ after admission in a subgroup of 56 patients (20.9\%). In these patients, median (IQR) copeptin levels decreased from $14.7(5.4-34.7)$ to $8.7(4.6-14.8) \mathrm{pmol} \cdot \mathrm{L}^{-1}$ after $24 \mathrm{~h}(\mathrm{p}<0.001$; fig. 2). All but three patients showed either a decrease of copeptin concentration after $24 \mathrm{~h}$ or had copeptin concentrations below the calculated optimal cut-off value. Two patients with increased copeptin levels after $24 \mathrm{~h}$ (patient 1: from 0.9 to $95.7 \mathrm{pmol} \cdot \mathrm{L}^{-1}$; patient 2: from 66.6 to $562.2 \mathrm{pmol} \cdot \mathrm{L}^{-1}$ ) had major bleedings (upper gastrointestinal and retroperitoneal, respectively) requiring emergency interventions (intubation and catecholamine administration) and blood transfusions.

\section{Prognostic value of copeptin with regard to study outcomes}

Within the first 30 days, 15 patients (5.6\%) reached the primary outcome (adverse 30-day outcome) and nine patients (3.4\%) reached the secondary outcome (all-cause 30-day death). More specifically, 11 patients needed catecholamine administration, 11 patients required mechanical ventilation, six patients underwent cardiopulmonary resuscitation and four patients died due to the initial pulmonary embolism. Patients who reached the primary outcome had higher copeptin levels on admission compared to patients with a favourable clinical course (median (IQR) 51.8 (21.6-90.8) versus 13.2 (5.9-39.3) $\mathrm{pmol} \cdot \mathrm{L}^{-1}$, $\mathrm{p}=0.020$ ). ROC analysis yielded an AUC of 0.68 for copeptin with regard to an adverse 30 -day outcome, which tended to be smaller compared to hsTnT $(0.72, \mathrm{p}=0.66)$ and NT-proBNP $(0.81, \mathrm{p}=0.070$; fig. 3). A copeptin concentration of $24 \mathrm{pmol} \cdot \mathrm{L}^{-1}$ was identified as the optimal cut-off value in our patient population. While the prognostic sensitivity of copeptin was lower, it was associated with a higher prognostic specificity if compared to hsTnT and NT-proBNP (cut-off values $14 \mathrm{pg} \cdot \mathrm{mL}^{-1}$ and $600 \mathrm{pg} \cdot \mathrm{mL}^{-1}$, respectively; table 2). Using univariable logistic regression analysis, copeptin levels $\geqslant 24 \mathrm{pmol} \cdot \mathrm{L}^{-1}$ were associated with a 5.4-fold increased risk for an adverse 30-day outcome (95\% CI 1.68-17.58; $\mathrm{p}=0.005)$. As shown in table 3, besides copeptin, diabetes mellitus, hypoxia, tachycardia and the ESC 2014 algorithm were identified as univariable predictors of the primary outcome. The highest odds ratio was observed for patients with copeptin $\geqslant 24 \mathrm{pmol} \cdot \mathrm{L}^{-1}$, hsTnT $\geqslant 14 \mathrm{pg} \cdot \mathrm{mL}^{-1}$ and NT-proBNP $\geqslant 600 \mathrm{pg} \cdot \mathrm{mL}^{-1}$. Importantly, the predictive value of copeptin $\geqslant 24 \mathrm{pmol} \cdot \mathrm{L}^{-1}$ remained independent after adjustment for each variable found to be associated with copeptin levels above the median (shown in online supplementary table S1).

Copeptin levels on admission did not differ in patients reaching the secondary outcome if compared to patients who survived the first 30 days $\left(23.4(5.9-38.9) \mathrm{pmol} \cdot \mathrm{L}^{-1}\right.$ versus $13.6(6.0-44.3) \mathrm{pmol} \cdot \mathrm{L}^{-1}$, $\mathrm{p}=0.695)$. Furthermore, neither copeptin $\geqslant 24 \mathrm{pmol} \cdot \mathrm{L}^{-1}(\mathrm{p}=0.585)$ nor exceeding the calculated optimal cut-off value for the secondary outcome of $19 \mathrm{pmol} \cdot \mathrm{L}^{-1}(\mathrm{p}=0.413)$ were associated with an increased risk for 30-day all-cause death.

Optimising risk assessment by integration of copeptin in a novel biomarker-based strategy When combining the three investigated biomarkers (hsTnT, NT-proBNP and copeptin), specificity and positive predictive value with regard to the primary study outcome reached 0.83 and 0.20 , respectively

FIGURE 2 Copeptin plasma levels on admission and after $24 \mathrm{~h}$ in a subgroup of 56 patients.








FIGURE 3 Receiver operating characteristic curve for copeptin, high-sensitivity troponin $\mathrm{T}$ (hsTnT) and $\mathrm{N}$-terminal pro-brain natriuretic peptide (NT-proBNP) with regard to an adverse 30-day outcome. AUC: area under the curve.

(table 2). Additionally, using a multivariable logistic regression model with backward stepwise selection including parameters univariably associated with an adverse 30-day outcome (shown in table 3), the final model consisted of copeptin $\geqslant 24 \mathrm{pmol} \cdot \mathrm{L}^{-1}(\mathrm{OR}, 4.13,95 \%$ CI $1.24-13.78 ; \mathrm{p}=0.021)$, hsTnT $\geqslant 14 \mathrm{pg} \cdot \mathrm{mL}^{-1}$ and NT-proBNP $\geqslant 600 \mathrm{pg} \cdot \mathrm{mL}^{-1}$ (odds ratios were not calculable for either of these). Therefore, we tested whether a novel stepwise biomarker-based strategy might help to optimise risk stratification of normotensive pulmonary embolism patients. Based on the excellent negative predictive value of hsTnT and NT-proBNP, we used these two biomarkers as the first step. As shown in figure $4 \mathrm{a}, 141$ patients $(52.8 \%)$ with hsTnT $<14 \mathrm{pg} \cdot \mathrm{mL}^{-1}$ or NT-proBNP $<600 \mathrm{pg} \cdot \mathrm{mL}^{-1}$ were classified as low risk. Importantly, none of them had an adverse 30-day outcome. In a second step, in patients with elevated hsTnT and NT-proBNP levels, copeptin $\geqslant 24 \mathrm{pmol} \cdot \mathrm{L}^{-1}$ was used to classify patients in an intermediate-low and an intermediate-high risk subgroup. Of $71(26.6 \%)$ patients stratified as intermediate-low risk, 5.6\% had an adverse 30-day outcome and of the 55 (20.6\%) patients stratified as intermediate-high risk, $20.0 \%$ had an adverse 30-day outcome.

Comparing this biomarker-based strategy with the ESC 2014 algorithm (fig. 4b), more patients were categorised as low risk (141 $(52.8 \%)$ out of 267 patients versus $47(17.5 \%)$ out of 268 patients; $\mathrm{p}<0.001)$

TABLE 2 Prognostic performances of laboratory biomarkers and risk assessment models with regard to an adverse 30 -day outcome

$\begin{array}{cccccc}\text { Sensitivity } & \text { Specificity } & \text { PPV } & \text { NPV } & \text { LR+ } & \text { LR- } \\ (95 \% \mathrm{CI}) & (95 \% \mathrm{CI}) & (95 \% \mathrm{CI}) & (95 \% \mathrm{CI}) & (95 \% \mathrm{CI}) & (95 \% \mathrm{CI})\end{array}$

\begin{tabular}{|c|c|c|c|c|c|c|}
\hline \multicolumn{7}{|l|}{ Laboratory biomarker } \\
\hline $\mathrm{hsTnT} \geqslant 14 \mathrm{pg} \cdot \mathrm{mL}^{-1}$ & $1.00(0.80-1.00)$ & $0.40(0.34-0.46)$ & $0.09(0.06-0.14)$ & $1.00(0.96-1.00)$ & $1.67(1.50-1.84)$ & \\
\hline $\mathrm{NT}-$ proBNP $\geqslant 600 \mathrm{pg} \cdot \mathrm{mL}^{-1}$ & $1.00(0.80-1.00)$ & $0.51(0.45-0.57)$ & $0.11(0.07-0.17)$ & $1.00(0.97-1.00)$ & $1.96(1.80-2.32)$ & \\
\hline Copeptin $\geqslant 24 \mathrm{pmol} \cdot \mathrm{L}^{-1}$ & $0.73(0.48-0.89)$ & $0.66(0.60-0.72)$ & $0.11(0.07-0.19)$ & $0.98(0.94-0.99)$ & $2.18(1.54-3.10)$ & $0.40(0.17-0.93)$ \\
\hline $\begin{array}{l}\text { Copeptin } \geqslant 24 \mathrm{pmol} \cdot \mathrm{L}^{-1} \text { and } \\
\text { hsTnT } \geqslant 14 \mathrm{pg} \cdot \mathrm{mL}^{-1} \text { and } \\
\mathrm{NT}-\text { proBNP } \geqslant 600 \mathrm{pg} \cdot \mathrm{mL}^{-1}\end{array}$ & $0.73(0.48-0.89)$ & $0.83(0.77-0.87)$ & $0.20(0.12-0.32)$ & $0.98(0.95-0.99)$ & $4.25(2.80-6.30)$ & $0.32(0.14-0.75)$ \\
\hline \multicolumn{7}{|l|}{ Risk assessment models } \\
\hline $\begin{array}{l}\text { ESC } 2014 \text { algorithm } \\
\text { (intermediate-high risk } \\
\text { versus low risk/intermediate-low risk) }\end{array}$ & $0.67(0.42-0.85)$ & $0.70(0.64-0.75)$ & $0.12(0.06-0.20)$ & $0.97(0.94-0.99)$ & $2.22(1.48-3.32)$ & $0.48(0.23-0.98)$ \\
\hline $\begin{array}{l}\text { Bova score (intermediate-high risk } \\
\text { versus low risk/intermediate-low risk) }\end{array}$ & $0.33(0.15-0.58)$ & $0.85(0.80-0.88)$ & $0.11(0.05-0.24)$ & $0.96(0.92-0.98)$ & $2.15(1.00-4,66)$ & $0.79(0.55-1.13)$ \\
\hline
\end{tabular}

Sensitivity, specificity, positive and negative predictive values and positive and negative likelihood ratios of dichotomised/dichotomous biomarkers, risk assessment models and combination of biomarkers with regard to an adverse 30-day outcome. PPV: positive predictive value; NPV: negative predictive value; LR+/-: positive/negative likelihood ratio; hsTnT: high-sensitivity troponin T; NT-proBNP: N-terminal pro-brain natriuretic peptide; ESC: European Society of Cardiology. 
TABLE 3 Predictors of an adverse 30-day outcome

OR $(95 \% \mathrm{CI})$

p-value

\section{Comorbidities}

Malignant tumour

Chronic cardiopulmonary disease

Diabetes mellitus

Renal insufficiency

$1.70(0.60-4.84)$

$3.26(1.10-9.62)$

$2.48(0.87-7.10)$

0.033

Symptoms and clinical status on admission

Syncope

Hypoxia

Tachycardia

$1.93(0.51-7.25)$

$3.85(1.28-11.60)$

$3.76(1.29-10.95)$

$2.08(0.43-9.99)$

Mild hypotension

$2.91(0.90-9.38)$

RV dysfunction on echocardiography or MDCT

Laboratory biomarkers

$\mathrm{hsTnT} \geqslant 14 \mathrm{pg} \cdot \mathrm{mL}^{-1}$

NT-proBNP $\geqslant 600 \mathrm{pg} \cdot \mathrm{mL}^{-1}$

Copeptin $\geqslant 24 \mathrm{pmol} \cdot \mathrm{L}^{-1}$

Copeptin $\geqslant 24 \mathrm{pmol} \cdot \mathrm{L}^{-1}$ and $\mathrm{hsTnT} \geqslant 14 \mathrm{pg} \cdot \mathrm{mL}^{-1}$ and NT-proBNP $\geqslant 600 \mathrm{pg} \cdot \mathrm{mL}^{-1}$

OR not calculable

$5.44(1.68-17.58)$

$13.00(3.91-42.72)$

Risk assessment models

ESC 2014 algorithm (intermediate-high risk versus low risk/intermediate-low risk)

$4.66(1.54-14.09)$

Bova score (intermediate-high risk versus low risk/intermediate-low risk)

Definitions are provided in the material and methods section of the online supplementary material. RV: right ventricular: MDCT: multidetector computed tomography; hsTnT: high-sensitivity troponin T; NT-proBNP: N-terminal pro-brain natriuretic peptide; ESC: European Society of Cardiology.

without additional adverse events in this subgroup. Furthermore, more patients classified as intermediatehigh risk had an adverse outcome compared to the intermediate-high risk group based on the ESC 2014 algorithm (20.0\% versus $11.6 \%$; fig. 4$)$.

Using the Bova score, 130 out of 267 (48.7\%) patients were classified as low risk and none of them reached the primary outcome. However, further stratification by the use of the Bova score did not help to fine-tune risk assessment: patients classified as intermediate-low risk (93 patients (34.8\%)) and intermediate-high risk (44 patients $(16.5 \%)$ ) had an almost identical rate of an adverse 30 -day outcome (10.8\% and $11.4 \%$, respectively).

\section{Discussion}

Copeptin: a novel biomarker in normotensive pulmonary embolism

The present study is the first demonstrating the prognostic importance of copeptin in normotensive patients with acute pulmonary embolism. Copeptin has been identified as a valuable biomarker for diagnosis and risk stratification in numerous cardiovascular conditions. For example, in patients with heart failure, copeptin was a valuable predictor of mortality [18, 19]. NICKEL et al. [20] demonstrated that elevated copeptin levels were independently associated with poor outcome in patients with pulmonary arterial hypertension. In agreement with these reports, in the present study, patients with chronic cardiopulmonary diseases presented more frequently with copeptin levels above the median. Copeptin levels have been found to be higher in patients with renal insufficiency [21] or diabetes mellitus [22]. Accordingly, in our study population we found more often copeptin levels above the median in patients with diabetes mellitus or with renal insufficiency (table 1). Of note, the median copeptin concentration of $14 \mathrm{pmol} \cdot \mathrm{L}^{-1}$ in the present patient population was identical to the optimal cut-off value calculated for patients with acute myocardial infarction (AMI) $[9,10]$.

Due to its fast release kinetics [7], copeptin appears to be particularly useful for prognostic assessment of acute diseases, e.g. in the emergency department [11]. In combination with hsTnT it allowed rapid and reliable exclusion of AMI at presentation $[9,10]$. Concordantly, in the present study all but three patients with initially elevated copeptin levels had a decrease in copeptin concentrations $24 \mathrm{~h}$ after admission (fig. 2). Two of the three cases with increased copeptin levels after $24 \mathrm{~h}$ were explained by major bleedings requiring emergency interventions or blood transfusions.

In our study, patients with an adverse 30-day outcome had higher copeptin levels on admission compared to patients with a favourable clinical course. Moreover, copeptin levels above the calculated optimal cut-off value of $24 \mathrm{pmol} \cdot \mathrm{L}^{-1}$ were associated with a 5.4 -fold increased risk for an adverse 30 -day outcome which 
a)

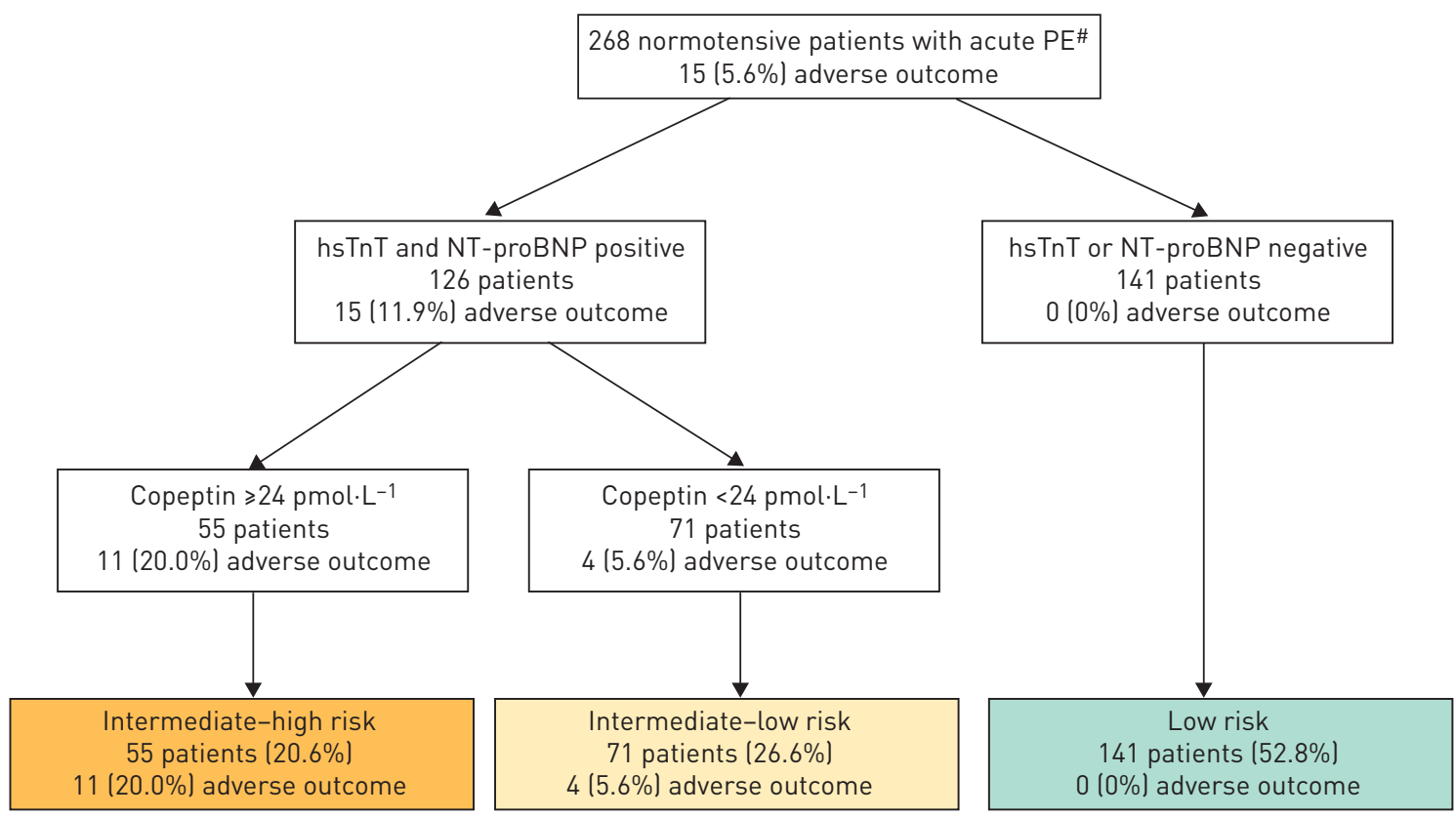

b)



FIGURE 4 Risk assessment strategies of normotensive patients with pulmonary embolism. a) Risk assessment with a new biomarker-based strategy based on N-terminal pro-brain natriuretic peptide (NT-proBNP), high-sensitivity troponin T (hsTnT) and copeptin. b) Risk assessment as proposed by the 2014 European Society of Cardiology (ESC) guideline. Number (\%) of patients with an adverse 30-day outcome are shown for each step. HsTnT levels $\geqslant 14 \mathrm{pg} \cdot \mathrm{mL}^{-1}$ and NT-proBNP levels $\geqslant 600 \mathrm{pg} \cdot \mathrm{mL}^{-1}$ were defined as positive. \#: in one patient with elevated hsTnT $\left(109.8 \mathrm{pg} \cdot \mathrm{mL}^{-1}\right.$ ) levels, NT-proBNP plasma concentrations could not be measured on admission. Thus, stratification to risk classes using the biomarker-based strategy was not possible and the patient was excluded. Of note, in four patients neither multidetector computed tomography nor an echocardiographic examination for assessment of right ventricular function was performed; three of them were classified as intermediatelow risk and one patient as low risk using the ESC 2014 algorithm shown in b). Definitions are provided in the material and methods section of the online supplementary material. PE: pulmonary embolism; SPESI: simplified Pulmonary Embolism Severity Index; RV: right ventricular.

remained independent in a multivariable logistic regression model using backward stepwise selection. However, elevated copeptin levels were not associated with an increased risk for all-cause death within 30 days. This finding indicates that copeptin, as a marker of the systemic response to impaired haemodynamics due to RV failure predicts pulmonary embolism-related complications in the acute phase 
of pulmonary embolism (which might require more aggressive treatment regimens) rather than providing general prognostic information (e.g. all-cause mortality) such as, for example, the biomarker growth-differentiation factor-15 [23].

\section{Risk stratification of normotensive pulmonary embolism}

The algorithm for risk assessment proposed by the current 2014 ESC guideline suggests the identification of low-risk patients based on a negative simplified Pulmonary Embolism Severity Index (sPESI) (in the absence of elevated cardiac biomarkers and absence of RV dysfunction on imaging procedures, if performed) [1]. Indeed, in our study population none of the patients in the ESC 2014 low-risk group reached the primary outcome. However, compared to previous cohort studies classifying approximately one-third of pulmonary embolism patients as low risk based on the sPESI [13, 24], in our study the proportion of patients as categorised as low risk by the ESC 2014 algorithm was as low as 17.5\% (fig. 4b). This might be explained, at least in part, by the fact that biomarker testing and assessment of RV function were performed as a part of the study protocol in all patients. Notably, out of $100(37.3 \%)$ patients with a sPESI of 0, one patient with an adverse 30-day outcome would have been missed without further testing.

At the other end of the risk spectrum, the PEITHO study demonstrated that in normotensive pulmonary embolism patients with elevated troponin levels and RV dysfunction on echocardiography or MDCT, the primary endpoint (death or haemodynamic decompensation within 7 days) was reduced by administration of thrombolysis (OR $0.44,95 \%$ CI $0.23-0.87 ; \mathrm{p}=0.015$ ) [17]. However, the complication rate in the placebo group was comparably low (28 (5.6\%) out of 499 patients) if compared to cohort studies. For example, Bova et al. [3] observed a 30-day pulmonary embolism-related complication rate of $29.2 \%$ for patients with a Bova score stage III (intermediate-high risk). In our study, patients classified as intermediate-high risk by the use of the Bova score and ESC 2014 algorithm had a similar rate of an adverse 30-day outcome (11.6\% and $11.4 \%$, respectively).

\section{A novel stepwise biomarker-based risk assessment strategy}

Risk assessment using the algorithm proposed by the 2014 ESC guideline requires three steps (assessment of haemodynamic stability, calculation of the sPESI and laboratory testing/imaging procedures), resulting in a complex and time-, person- and potentially cost-intensive approach. Although our study was performed at a university hospital, only 157 (58.6\%) patients received an echocardiographic examination within $48 \mathrm{~h}$, demonstrating the limited availability (which might be even greater in smaller hospitals). However, this might also be caused by the more frequent use of MDCT for evaluation of RV (dys-) function in our institution considering the subjective observer-related nature of echocardiographic findings and the lack of a validated definition of RV dysfunction. Given these limitations of the ESC 2014 algorithm, we developed a novel stepwise biomarker-based strategy which might help to optimise risk stratification of normotensive pulmonary embolism.

As shown in previous cohorts [12-14], in the present study hsTnT and NT-proBNP yielded an excellent negative predictive value $\left(100 \%\right.$ each). Consequently, we used hsTnT $<14 \mathrm{pg} \cdot \mathrm{mL}^{-1}$ or NT-proBNP $<600 \mathrm{pg} \cdot \mathrm{mL}^{-1}$ to identify low-risk patients. Importantly, by this approach as many as $52.8 \%$ of the patients were categorised as low risk and none of them reached the primary outcome. Thus, the biomarker-based strategy appeared to be superior for the identification of normotensive pulmonary embolism patients at low risk compared to ESC 2014 algorithm [1] (52.8\% without adverse outcome versus 17.5\% without adverse outcome, $\mathrm{p}<0.001)$. In a second step, in patients with elevated hsTnT and NT-proBNP levels, copeptin $\geqslant 24 \mathrm{pmol} \cdot \mathrm{L}^{-1}$ was used to identify $55(20.6 \%)$ patients at intermediate-high risk. The rate of an adverse 30-day outcome in this group was nearly doubled compared to the ESC 2014 algorithm [1] or Bova score [3] (20.0\% versus $11.6 \%$ versus $11.4 \%$, respectively). Additionally, the Bova score failed to select the patient subgroup at highest risk: patients classified as intermediate-low and intermediate-high risk had an almost identical rate of an adverse 30 -day outcome $(10.8 \%$ and $11.4 \%$, respectively). Thus, the biomarker-based strategy appeared to be superior for the identification of normotensive pulmonary embolism patients at intermediate-high risk who might be candidates for thrombolytic therapy. However, given the high bleeding rate (especially intracranial) in the PEITHO study [17], further efforts are warranted to determine the best strategy for identification of intermediate-high risk pulmonary embolism patients with a low bleeding risk in whom thrombolysis appears to be safe.

\section{Limitations}

Some potential limitations of our study should be mentioned. First, although the number of patients with an adverse 30-day outcome was low given the single-centre study design, the rate of an adverse 30-day outcome (5.6\%) was comparable to other cohort studies and trials $[3,12,13,17,24]$. Second, the cut-off value of copeptin and the biomarker-based strategy were derived from the present patient cohort. Thus, external validation of the present study findings in a multicentre cohort is necessary to confirm the 
usefulness of copeptin and the novel simple biomarker-based strategy for risk stratification of normotensive pulmonary embolism.

\section{Conclusions}

Pending external validation, copeptin might be a useful novel biomarker reflecting the systemic response to impaired haemodynamics due to RV failure and thus a new pathophysiological axis for risk stratification of normotensive patients with acute pulmonary embolism. A novel simple biomarker-based strategy based on hsTnT, NT-proBNP and copeptin classified more patients as low risk, none of whom reached the primary outcome, and more patients in the intermediate-high-risk group had an adverse outcome compared to the algorithm proposed by the 2014 ESC guideline and the Bova score.

\section{Acknowledgements}

We thank Irene Schmidtmann (Institute for Medical Biostatistics, Epidemiology and Informatics (IMBEI), University Medical Centre Mainz, Johannes Gutenberg-University Mainz, Mainz, Germany) for expert statistical advice.

\section{References}

1 Konstantinides SV, Torbicki A, Agnelli G, et al. 2014 ESC guidelines on the diagnosis and management of acute pulmonary embolism. Eur Heart J 2014; 35: 3033-3069.

2 Konstantinides S, Torbicki A. Management of venous thromboembolism: an update. Eur Heart J 2014; 35 : 2855-2863.

3 Bova C, Sanchez O, Prandoni $\mathrm{P}$, et al. Identification of intermediate-risk patients with acute symptomatic pulmonary embolism. Eur Respir J 2014; 44: 694-703.

4 Fernández C, Bova C, Sanchez O, et al. Validation of a model for identification of patients at intermediate to high risk for complications associated with acute symptomatic pulmonary embolism. Chest 2015; 148: 211-218.

5 Wood KE. Major pulmonary embolism: review of a pathophysiologic approach to the golden hour of hemodynamically significant pulmonary embolism. Chest 2002; 121: 877-905.

6 Bolignano D, Cabassi A, Fiaccadori E, et al. Copeptin (CTproAVP), a new tool for understanding the role of vasopressin in pathophysiology. Clin Chem Lab Med 2014; 52: 1447-1456.

7 Liebetrau C, Nef H, Szardien S, et al. Release kinetics of copeptin in patients undergoing transcoronary ablation of septal hypertrophy. Clin Chem 2013; 59: 566-569.

8 Khan SQ, Dhillon OS, O’Brien RJ, et al. C-terminal provasopressin (copeptin) as a novel and prognostic marker in acute myocardial infarction: Leicester Acute Myocardial Infarction Peptide (LAMP) study. Circulation 2007; 115: 2103-2110.

9 Reichlin T, Hochholzer W, Stelzig C, et al. Incremental value of copeptin for rapid rule out of acute myocardial infarction. J Am Coll Cardiol 2009; 54: 60-68.

10 Maisel A, Mueller C, Neath SX, et al. Copeptin helps in the early detection of patients with acute myocardial infarction: primary results of the CHOPIN trial (copeptin helps in the early detection of patients with acute myocardial infarction). J Am Coll Cardiol 2013; 62: 150-160.

11 Nickel CH, Bingisser R, Morgenthaler NG. The role of copeptin as a diagnostic and prognostic biomarker for risk stratification in the emergency department. BMC Med 2012; 10: 7.

12 Lankeit M, Friesen D, Aschoff J, et al. Highly sensitive troponin T assay in normotensive patients with acute pulmonary embolism. Eur Heart J 2010; 31: 1836-1844.

13 Lankeit M, Jiménez $\mathrm{D}$, Kostrubiec $\mathrm{M}$, et al. Predictive value of the high-sensitivity troponin $\mathrm{T}$ assay and the simplified Pulmonary Embolism Severity Index in hemodynamically stable patients with acute pulmonary embolism: a prospective validation study. Circulation 2011; 124: 2716-2724.

14 Lankeit M, Jiménez $\mathrm{D}$, Kostrubiec $\mathrm{M}$, et al. Validation of $\mathrm{N}$-terminal pro-brain natriuretic peptide cut-off values for risk stratification of pulmonary embolism. Eur Respir J 2014; 43: 1669-1677.

15 Dellas C, Puls M, Lankeit M, et al. Elevated heart-type fatty acid-binding protein levels on admission predict an adverse outcome in normotensive patients with acute pulmonary embolism. J Am Coll Cardiol 2010; 55: $2150-2157$.

16 Torbicki A, Perrier A, Konstantinides S, et al. Guidelines on the diagnosis and management of acute pulmonary embolism: the Task Force for the Diagnosis and Management of Acute Pulmonary Embolism of the European Society of Cardiology (ESC). Eur Heart J 2008; 29: 2276-2315.

17 Meyer G, Vicaut E, Danays T, et al. Fibrinolysis for patients with intermediate-risk pulmonary embolism. $N$ Engl J Med 2014; 370: 1402-1411.

18 Neuhold S, Huelsmann M, Strunk G, et al. Comparison of copeptin, B-type natriuretic peptide, and amino-terminal pro-B-type natriuretic peptide in patients with chronic heart failure: prediction of death at different stages of the disease. J Am Coll Cardiol 2008; 52: 266-272.

19 Voors AA, von Haehling S, Anker SD, et al. C-terminal provasopressin (copeptin) is a strong prognostic marker in patients with heart failure after an acute myocardial infarction: results from the OPTIMAAL study. Eur Heart $J$ 2009; 30: 1187-1194.

20 Nickel NP, Lichtinghagen R, Golpon $\mathrm{H}$, et al. Circulating levels of copeptin predict outcome in patients with pulmonary arterial hypertension. Respir Res 2013; 14: 130.

21 Meijer E, Bakker SJ, de Jong PE, et al. Copeptin, a surrogate marker of vasopressin, is associated with accelerated renal function decline in renal transplant recipients. Transplantation 2009; 88: 561-567.

22 Enhörning S, Wang TJ, Nilsson PM, et al. Plasma copeptin and the risk of diabetes mellitus. Circulation 2010; 121: $2102-2108$.

23 Lankeit M, Kempf T, Dellas C, et al. Growth differentiation factor-15 for prognostic assessment of patients with acute pulmonary embolism. Am J Respir Crit Care Med 2008; 177: 1018-1025.

24 Jiménez D, Aujesky D, Moores L, et al. Simplification of the pulmonary embolism severity index for prognostication in patients with acute symptomatic pulmonary embolism. Arch Intern Med 2010; 170: 1383-1389. 